Case Reports

\title{
Bilateral Renal Cortical Abscesses Presenting as Pyrexia of Unknown Origin in an Immunocompetent Individual
}

\author{
Anupam Sarkar, Sandipan Banik, Debashis Sadhukhan, Prabir Kumar Kundu, \\ Indira Maisnam, Aniruddha Ray and Apurba Kumar Mukherjee
}

Department of General Medicine, RG Kar Medical College and Hospital 1, Kshudiram Bose Sarani, Kolkata-70004, India

Article history

Received: 19-10-2014

Revised: $15-11-2014$

Accepted: 28-04-2015

Corresponding Author: Indira Maisnam

Department of General

Medicine, RG Kar Medical

College and Hospital 1,

Kshudiram Bose Sarani,

Kolkata-70004, India

Email: i.maisnam@gmail.com

\begin{abstract}
The diagnosis of renal cortical abscess can be missed as the condition is frequently associated with blood and urine culture negativity. Moreover, imaging by ultrasonography in the early stages of the disease may miss small or developing abscesses and carbuncles. Renal abscesses being more common in the immunosuppressed, the condition may be overlooked in the immunocompetent. We report a case of bilateral renal cortical abscesses in an immunocompetent individual who presented to us with Pyrexia of Unknown Origin (PUO). The patient's urine and blood culture were negative and initial ultrasonography of the abdomen revealed no abnormality. Based on clinical suspicion a repeat ultrasonography was done, which detected bilateral renal cortical abscesses. Thus, when in doubt repeat or more sensitive imaging is required for correct diagnosis. Important differences exist between renal cortical and cortico-medullary abscesses and this awareness guides therapeutic decision. Missing a renal cortical abscess based on single negative imaging could be catastrophic.
\end{abstract}

Keywords: Renal Cortical Abscess, Immune-Competent, PUO

\section{Introduction}

Bilateral renal cortical abscesses occur as a result of hematogenous seeding from infected foci elsewhere in the body. It is easy to miss this condition as the urine and blood culture are frequently negative, imaging studies may not be suggestive in initial stages, the inciting focus may not be obvious at the time of illness and they rarely occur in the immunocompetent host. We report such a case in an immunocompetent individual with the aim to increase awareness of this condition and emphasize that it may present as pyrexia of unknown origin. It is easy but dangerous to miss this condition.

\section{Case Report}

\section{Brief History, Clinical Findings and Investigations}

A 26 year old previously healthy man came with the chief complaints of high grade fever, abdominal pain and vomiting for the past two weeks. The fever was continuous, high grade and associated with chills. Abdominal pain was deep aching in nature with radiation to back. The patient denied any history of chronic excessive alcohol intake or use of any illicit drugs. He did not have any dysuria; or any cough and expectoration. There was no joint pain or rash or similar episode in the past. He was married and had a single sexual partner.

One week prior to attending our Hospital he had undergone a few investigations which had revealed neutrophilic leucocytosis (Table 1). His peripheral blood for malarial parasite was negative. The urine examination and ultrasonography of the abdomen were normal. He had received a course of tablet azithromycin with no respite from his symptoms. He was referred to our Hospital with a diagnosis of Pyrexia of Unknown Origin (PUO).

On examination the patient had high grade fever $\left(102.6^{\circ} \mathrm{F}\right)$. He was mildly anemic and there was no jaundice. He was conscious but was severely distressed by the abdominal pain. The abdomen was soft and there was tenderness over the right hypochondrium and right lumbar region. The right renal angle was very tender. The left renal angle and the hypogastrium were nontender. There was no organomegaly, no palpable mass and no fluid in the abdomen. The bowel sounds were clear. Examination of the respiratory system revealed a stony dullness and diminished breathe sounds in the right infra-mammary, infra-axillary and infra-scapular regions suggestive of a right sided mild-moderate pleural effusion. Examination of the cardiovascular system was normal and there was no evidence of any murmur. The 
skin and mucous membrane looked healthy and there was no evidence of any infection, rash, ulcer or bleeding. His dental hygiene was average. Examination of other systems revealed no abnormality. A clinical diagnosis of right upper urinary tract infection (possibly right sided pyelonephritis) was made. Blood and urine were sent for routine investigation including culture. Patient was immediately started with intravenous ceftriaxone $2 \mathrm{~g}$ once daily pending investigation reports.

Investigations in our Hospital revealed neutrophilic leucocytosis with toxic granules without any abnormal cells in the peripheral blood. Urine routine examination was essentially normal. Blood and urine culture were negative. Other relevant investigations including plasma glucose and ELISA for HIV were negative (Table 1).

Despite the negative findings on ultrasonography done 5 days back, a repeat ultrasonography of the Kidney, Ureter and Bladder (KUB) region was done as there were significant findings suggestive of urinary tract involvement on clinical examination. Ultrasonography showed a hypoechoic space occupying lesion measuring $3.6 \mathrm{~cm}$ in maximum diameter occupying part of the right middle and lower pole of the right kidney beneath the cortex. Another hypoechoic subcortical lesion was seen in the mid-pole of the left kidney measuring $\sim 2 \mathrm{~cm}$ in maximum diameter. CT scan of the abdomen with contrast showed a round hypodense lesion measuring $33 \times 32 \mathrm{~mm}$ with peripheral rim enhancement in the anterior cortex of right renal lower pole (Fig. 1). Another smaller hypodense lesion measuring $23 \times 20 \mathrm{~mm}$ was seen in the postero-lateral cortex at right mid-pole. Left kidney showed an oval hypodense lesion measuring $23 \times 18 \mathrm{~mm}$ in the postero-medial aspect of mid pole near the cortex. The findings were suggestive of bilateral renal cortical abscesses. There were no renal calculi or structural anomaly of the urinary tract on imaging. Fine needle aspiration of the abscess was not done as the patient did not provide consent. Chest X Ray showed a right-sided mild pleural effusion. The pleural fluid was transudative in character. Echocardiography was normal without any vegetations on the heart valves or endocardium. A diagnosis of bilateral renal cortical abscesses with right sided transudative pleural effusion in an immunocompetent individual was made.

\section{Course in the Hospital}

The patient continued to be febrile even after $24 \mathrm{~h}$ of admission. Intravenous Linezolid 600mg twice daily was added for coverage of gram-positive cocci. However, the patient remained febrile $48 \mathrm{~h}$ after starting linezolid. His hemogram after $48 \mathrm{~h}$ of admission showed no improvement (Table 2). Finally, it was decided to switch over to a broader spectrum antibiotic as the patient continued to be febrile. Injection Meropenem $1 \mathrm{~g}$ thrice daily was given and other antibiotics were stopped.
Within $48 \mathrm{~h}$ of the first dose of meropenem the patient became afebrile and his blood counts had improved (Table 2). Meropenem was continued for three weeks.

Over the ensuing weeks, resolution of the abscess was seen on serial ultrasonography. CT scan of the abdomen done at 7 weeks showed complete resolution of the abscess (Fig. 2).

Table 1. Showing haematological and biochemical parameters of the patient before and at the time of admission

\begin{tabular}{lcr}
\hline Parameter & Before admission & At admission \\
\hline Hb (g/dL) & 13.6 & 12.20 \\
TLC (per mL) & 16200.0 & 19600.00 \\
PMN (\%) & 74.00 & 78.00 \\
Lymphocytes (\%) & 22.00 & 16.00 \\
Monocytes (\%) & 2.00 & 4.00 \\
Platelets (per mL) & 220000.00 & 260000.00 \\
ESR (mm) & 35.00 & 46.00 \\
Urea (mg/dL) & 32.00 & 34.00 \\
Creatinine (mg/dL) & 0.86 & 0.94 \\
Sodium (mmol/L) & 136.00 & 134.00 \\
Potassium (mmol/L) & $4,2.00$ & 4.10 \\
FPG (mg//dL) & 86.00 & 90.00 \\
Lipase (U/L) & & 16.00 \\
Amylae (U/L) & & 34.00 \\
ALT (U/L) & & 24.00 \\
AST (U/L) & 26.00 \\
ALP (U?L) & & 245.00 \\
Bilirubin (mg/dL) & & 0.90 \\
Albumin (mg/dL) & & 3.80 \\
ELISA for HIV & & negative \\
Urine routine & & 2-3 pus cells/HPF \\
Blood culture & & negative at $48 \mathrm{~h}$ \\
Urine culture & & negative at $48 \mathrm{~h}$
\end{tabular}

ALP: Alkaline Phosphatase, ALT: Alanine Transaminase, AST: Aspartate transaminase, ELISA: Enzyme-linked immunosorbent assay, ESR: Erythrocyte Sedimentation Rate, FPG: Fasting plasma glucose, Hb: Hemoglobin, HIV: Human Immunodeficiency Virus, TLC: Total Leucocyte Count

Table 2. Showing haematological, biochemical parameters including urine and blood culture of the patient at various stages in the course of the illness

\begin{tabular}{lrr}
\hline Parameter & $48 \mathrm{~h}(\mathrm{~A})$ & $48 \mathrm{~h}(\mathrm{M})$ \\
\hline $\mathrm{Hb}(\mathrm{g} / \mathrm{dL})$ & 11.8 & 11.0 \\
TLC (per mL) & 21400.00 & 13000.0 \\
PMN (\%) & 80.00 & 74.0 \\
Lymphocytes (\%) & 15.00 & 25.0 \\
Monocytes (\%) & 3.00 & 2.0 \\
Platelets (per mL) & 250000.00 & 230000.0 \\
ESR (mm) & 40.00 & 36.0 \\
Urea (mg/dL) & 32.00 & 28.0 \\
Creatinine (mg/dL) & 0.90 & 0.87 \\
Sodium (mmol/L) & 132.00 & 136.0 \\
Potassium (mmol/L) & $4,3.00$ & 4.2 \\
FPG (mg//dL) & 88.00 & \\
\hline
\end{tabular}

$48 \mathrm{~h}$ (A): After $48 \mathrm{~h}$ of admission; $48 \mathrm{~h}$ (M): After $48 \mathrm{~h}$ of injection meropenem., ESR: Erythrocyte Sedimentation Rate, FPG: Fasting Plasma Glucose, Hb: Hemoglobin, TLC: Total leucocyte count 


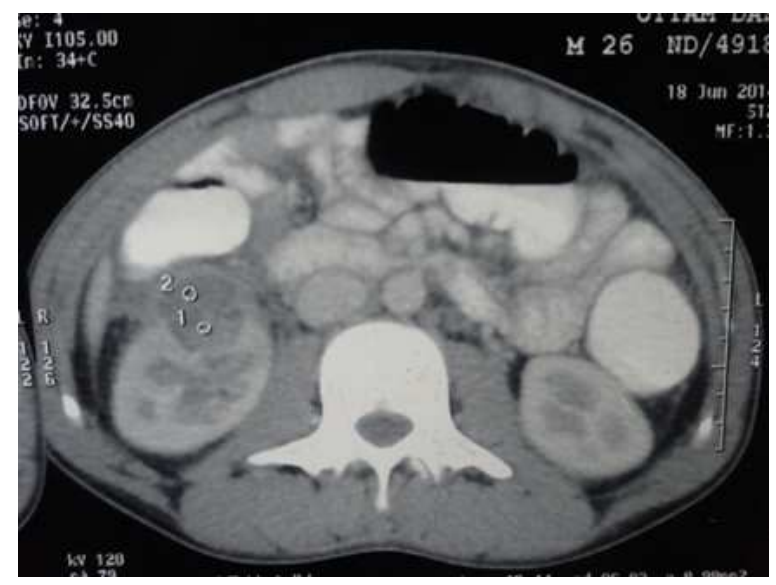

Fig. 1. Contrast CT scan of the kidneys showing 2 cortical abscesses in the right kidney

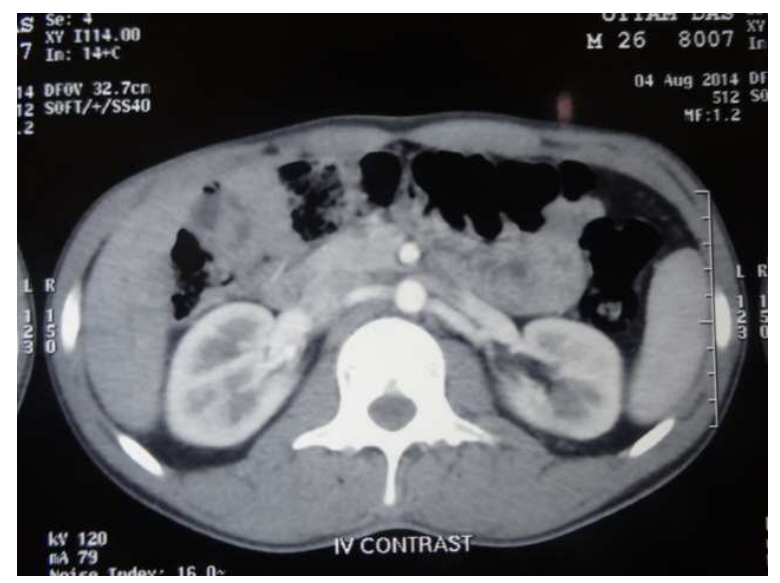

Fig. 2. CT scan of the kidneys done after 7 weeks showing complete resolution of the abscesses

\section{Discussion}

Renal and peri-renal abscess can occur as a complication of urinary tract infection or due to metastatic spread from a focus of infection elsewhere in the body. Renal abscess that occurs due to ascending infection from a lower urinary tract infection involves the medulla and are thus cortico-medullary abscesses (Foxman and Brown, 2003). Hematogenous seeding from foci of infection elsewhere in the body usually involve the cortex and are thus renal cortical abscesses, also known as renal carbuncle (Patterson and Andriole, 1987). Renal cortical abscesses are more common in men in the 2nd and 3rd decades (Moore and Gangai, 1967). Among the two, renal cortico-medullary abscesses are more common. Cortico-medullary abscesses commonly occur in patients with underlying risks like renal calculi, genito-urinary reflux disease or in immunocompromised and other high risk conditions like human immunodeficiency virus infection and diabetes mellitus (Johnson, 2003). The renal cortical abscess occur as a result of hematogenous seeding from skin and soft tissue, dental infection and infected heart valves and underlying immunosuppression increase the chance of renal cortical abscesses and their complication (Saiki et al., 1982). Both renal cortical abscess and cortico-medullary abscess can spread to the perinephric tissue and cause peri-nephric abscess.

There are certain similarities and important differences between the two conditions. Both are characterised by high grade fever with chills and both conditions can cause pain and tenderness in the renal angle. However, urinary symptoms of dysuria, increased urinary frequency and renal colic is frequently present in the renal cortico-medullary abscess, they are mostly absent in renal cortical abscess. Renal cortical abscess is mostly characterised by absent or few pus cells on urine routine microscopy and a negative urine culture, as was seen in our patients. However, some but not all patients may have a positive blood culture. The possible reasons for a negative blood culture are prior use of antibiotics, lack of persistent bacteremia or a bacteremia in the recent past which has now resolved. Symptoms of renal cortical abscess can develop after the inciting infection (e.g., skin infection) is no longer obvious. This is because it takes time for the abscess to develop and cause clinical symptoms after seeding. Another very important difference between the two conditions is the causative micro-organisms and thus the choice of antimicrobial therapy. Most renal cortico-medullary abscesses are cause by gram negative bacilli whereas staphylococcus aureus has been implicated as the etiologic agent in renal cortical abscesses (Dembry and Andriole, 1997). The diagnosis of renal cortical abscess can thus be missed in view of the frequently negative urine culture and rarely positive blood culture. Hence imaging studies are a must in the diagnosis of this condition. It must be kept in mind that ultrasound at the initial stages of the disease maybe negative as findings may be missed or may not be obvious at the early carbuncle stage. Hence repeat ultrasound or more sensitive imaging like CT scan should be done when in doubt (Cheng et al. 2008). Late diagnosis can be catastrophic with spread and rupture of the abscess. Imaging features of renal abscess consists of a hypoechoic mass on ultrasound or a well-defined lowdensity mass with a post-contrast enhancement of abscess wall on CT scan (Demertzis and Menias, 2007). Renal cortical abscess unless complicated is confined to the cortical and subcortical area, whereas renal corticomedullary abscess shows involvement of the medulla, with calyceal involvement and distortion. However, in advanced and complicated stages, due to expansion and rupture it may be not possible to distinguish the two (Yen et al., 1999). Ultrasonographic guided aspiration of the abscess and examining the aspirate for gram stain and culture identifies the etiologic agent. But where 
aspiration is not possible due to other co-morbid conditions or patient's refusal (as in our case); knowledge about the differences between renal corticomedullary abscess and renal cortical abscess can help us make an intelligent choice of antibiotics. Thus in our patient when the diagnosis of renal cortical abscess was made we added linezolid for staphylococcus aureus coverage. However, when the patient remained febrile without any improvement in the hematologic parameter, we converted to injection meropenem. Meropenem is a broad-spectrum antibiotic with wide coverage against many gram positive and negative bacteria. Considering the possibility that other gram positive-cocci and even gram-negative bacilli could have been the cause of the inciting focus (which was no longer obvious when the patient reported to us), we replaced injection ceftriaxone and linezolid with meropenem. We saw a marked improvement with meropenem with the patient becoming afebrile within $48 \mathrm{~h}$ of starting it. CT scan of the abdomen was done to determine the extent of the involvement and lesions that could have been missed on ultrasonography. Monitoring of the patient was done with clinical and haematological response and a serial ultrasonography. Serial ultrasonography helped us to monitor the decreasing size of the abscess and rule out peri-nephric or even medullary extension. Patient was put on injection meropenem for 3 weeks and the final CT scan showed complete disappearance of all the abscesses.

\section{Conclusion}

The following conclusions can be learnt from the above case: (i) Renal cortical abscess can be a cause of pyrexia of unknown origin even in an immunocompetent host; (ii) urine and blood culture are frequently negative; (iii) initial imaging may miss findings on ultrasonography and hence when in doubt repeat imaging should be done; (iv) causative organisms are different between renal cortical and cortico-medullary abscess and this awareness guides intelligent choice of antibiotics in conditions where aspiration and culture are not possible; (iv) serial ultrasonography is a suitable tool for non-invasive monitoring of abscesses.

The limitation of our report is that aspiration and culture which is the investigation of choice could not be done in our patient as unfortunately he did not provide consent. However, the choice of antibiotic was a scientific one and not done blindly, as it was chosen keeping in mind the usual organisms that would cause renal cortical abscesses. Such a decision for treatment may sometimes be required in a patient who has too small an abscess to be aspirated.

\section{Acknowledgement}

To the patient for his consent to publish this report and support staff of our department.

\section{Author Contributions}

Anupam Sarkar: Directly involved in patient treatment. Actively involved in administering treatment and arranging necessary investigations. Collected clinical photographs and wrote the manusript with Indira Maisnam

Sandipan Banik: Directly involved in patient treatment. Actively involved in administering treatment and arranging necessary investigations

Debashis Sadhukhan: Directly involved in patient treatment. Actively involved in administering treatment and arranging necessary investigations

Prabir Kumar Kundu: Directly involved in patient treatment. Provided clinical and scientific guidance

Indira Maisnam: Directly involved in patient treatment. Provided clinical and scientific guidance Collected clinical photographs and wrote the manusript with Anupam Sarkar

Aniruddha Ray: Directly involved in patient treatment. Provided clinical and scientific guidance

Apurba Kumar Mukherjee: Directly involved in patient treatment. Provided clinical and scientific guidance

\section{References}

Cheng, C.H., M.H. Tsai, L.H. Su, C.R. Wang and W.C. Lo et al., 2008. Renal abscess in children: A 10-year clinical and radiologic experience in a tertiary medical center. Pediatr Infect Dis. J., 27: 1025-1027. DOI: $10.1097 /$ INF.0b013e31817b617b

Dembry, L.M. and V.T. Andriole, 1997. Renal and Perirenal abscesses. Infect Dis. Clin North Am., 11: 663-680. PMID: 9378929

Demertzis, J. and C.O. Menias, 2007. State of the art: Imaging of renal infections. Emerg Radiol., 14: 13-22. PMID: 17318482

Foxman, B. and P. Brown, 2003. Epidemiology of urinary tract infections: Transmission and risk factors, incidence and cost. Infect. Dis. Clin. North Am. 17: 227-241. PMID: 12848468

Johnson, J.R., 2003. Microbial virulence determinants and the pathogenesis of urinary tract infection. Infect Dis. Clin. North Am., 17: 261-268. PMID: 12848470

Moore, C.A. and M.P. Gangai, 1967: Renal cortical abscess. J. Urol., 98: 303-306.

Patterson, J.E. and V.T. Andriole, 1987. Renal and perirenal abscesses. Infect Dis. Clin. North Am., 1: 907-926.

Saiki, J., N.D. Vaziri and C. Barton, 1982. Perinephric and intranephric abscesses: A review of the literature. West J. Med., 136: 95-102. PMID: 7039139

Yen, D.H., S.C. Hu, J. Tsai, W.F. Kao and C.H. Chern et al., 1999. Renal abscess: Early diagnosis and treatment. Am. J. Emerg. Med., 17: 192-197. PMID: 10102326 